Supporting Information

For

\title{
In Vitro Selection of RNA Aptamers Binding to Nanosized DNA for Constructing Artificial Riboswitches
}

\author{
Atsushi Ogawa* and Yu Itoh \\ Proteo-Science Center, Ehime University, 3 Bunkyo-cho, Matsuyama, Ehime 790-8577, Japan
}

\section{CONTENTS:}

Sequences of Synthetic Nucleic Acids. (p. S2)

Sequence of pTheo5-PSIV-IRES. (p. S4)

Sequences of Artificial Riboswitches. (p. S7)

Supplementary Figures and Tables. (p. S8) 


\section{Sequences of Synthetic Nucleic Acids.}

(written from 5' (left) to 3' (right); shaded: T7 promoter; underlined: mutated nucleotides; italic: additional nucleotides; bold: SpeI site)

Target nDNA: GGTTGC

M1: $\underline{\text { AGTTGC }}$

M2: GㅅTTGC

M3: AGGTTGC

nRNA: GGUUGC

FP1: ACG GCC AGT GAA TTG ACG CT

RP1: GAA GTT GAG TTG ATT GGA CGN NNN NNN NNN NNN NNN NNN NNN NNN NNN

NNC GTC CTG GGA AAC CGT TGT GGT CTC CCT ATA GTG AGT CGT ATT AC

T1: ACG GCC AGT GAA TTG ACG CTT ATT GGG ATG GAA CGT AAT ACG ACT CAC TAT

AGG GAG A

FP2: GTA ATA CGA CTC ACT ATA GGG AGA CCA CAA CGG TTT CCC AGG ACG

RP2: GAA GTT GAG TTG ATT GGA CG

FP3: CCA TCT CAT CCC TGC GTG TCT CCG ACT CAG ACC ACA ACG GTT TCC CAG GAC G

RP3: CCT CTC TAT GGG CAG TCG GTG ATG AAG TTG AGT TGA TTG GAC G

FP4: GTA ATA CGA CTC ACT ATA GG

RP4: TGG ACG GGT AAT GGG TT

T-6768: ACC ACA ACG GTT TCC CAG GAC GCG AGC CGG AAA CAT ACG GAG GCA ACC CTT GCG TCC AAT CAA CTC AAC TTC

T-1974: ACC ACA ACG GTT TCC CAG GAC GGT TGG TGC AAC CCG CCA GAT ATG GTG CAA CCG TCC AAT CAA CTC AAC TTC 
T-850: ACC ACA ACG GTT TCC CAG GAC GTG CTC CTT TTG CAA CCA GGA GAG CAG CCC GCG TCC AAT CAA CTC AAC TTC

T-547: ACC ACA ACG GTT TCC CAG GAC GGA TGC AAC CGT AAT GAC GCA ACC CAT TAC CCG TCC AAT CAA CTC AAC TTC

T-130: ACC ACA ACG GTT TCC CAG GAC GGA TAC GCA ACC CAC TCC GCA ACC AGT GGT CCG TCC AAT CAA CTC AAC TTC

T-C1: ACC ACA ACG GTT TCC CAG GAC GAA AAA AAA AAA AAA AAA AAA AAA AAA AAA ACG TCC AAT CAA CTC AAC TTC

T-C2: ACC ACA ACG GTT TCC CAG GAC GAA AAA AAA AAA AGC AAC CAA AAA AAA AAA ACG TCC AAT CAA CTC AAC TTC

T-C3: ACC ACA ACG GTT TCC CAG GAC GGC AAC CGC AAC CGC AAC CGC AAC CGC AAC CCG TCC AAT CAA CTC AAC TTC

T-547-m1: ACC ACA ACG GTT TCC CAG GAC GGA TGC TTC CGT AAT GAC GCA ACC CAT TAC CCG TCC AAT CAA CTC AAC TTC

T-547-m2: ACC ACA ACG GTT TCC CAG GAC GGA TGC AAC CGT AAT GAC GCI TCC CAT TAC CCG TCC AAT CAA CTC AAC TTC

T-547-m3: ACC ACA ACG GTT TCC CAG GAC GGA TGC TTC CGT AAT GAC GCT $\underline{\text { TCC CAT }}$ TAC CCG TCC AAT CAA CTC AAC TTC

T-547-mini: GTA ATA CGA CTC ACT ATA GGA CGG ATG CAA CCG TAA TGA CGC AAC CCA TTA CCC GTC CA

FP-P-1: GAA ATT AAT ACG ACT CAC TAT AGG GAG ACC ACA ACG GTT TCC CTG ACT ATG TGA TCT TAT TAA AAT TAG G

RP-PI: TTC CAT ACT AGT TTC TTT TTC TTG AAG TGA GAT TCT TTT CGC AC FP-5F: GAA ATT AAT ACG ACT CAC TAT AG 
FP-NL: GAT ATC ACT AGT AGG GTC TTC ACA CTC GAA GAT TTC

RP-3U-1: TCC CGA CTG GAA AGC GGG CAG TGA AAG GAA GGC CCA TGA GGC CCA GGA GCT CGA GTT ACG CCA GAA TGC GTT CGC ACA G

RP-3U-2: ATA CGC AAG GAA ACA GCT ATG ACC ATG TTA ATG CAG CTG GCA CGA CAG GTT TCC CGA CTG GAA AGC GGG CAG TGA A

FP-F: GAA ATT AAT ACG ACT CAC TAT AGG GAG ACC ACA ACG GT

RP-F: ATA CGC AAG GAA ACA GCT ATG AC

FP-NX-1: TCT ATG CAA CCG TAA TGA CGC AAC CCA TTA CAG ACC TCT GCT GAC TAT GTG

FP-N3-2: TAA TAC GAC TCA CTA TAG GGA GAC CAC AAC GGT TTC CCA CAT AGA CAA GAG GTC TAT GCA ACC GTA ATG ACG CAA C

FP-N4-2: TAA TAC GAC TCA CTA TAG GGA GAC CAC AAC GGT TTC CCA GCA TAG ACA AGA GGT CTA TGC AAC CGT AAT GAC GCA AC

FP-N5-2: TAA TAC GAC TCA CTA TAG GGA GAC CAC AAC GGT TTC CCA TGC ATA GAC AAG AGG TCT ATG CAA CCG TAA TGA CGC AAC

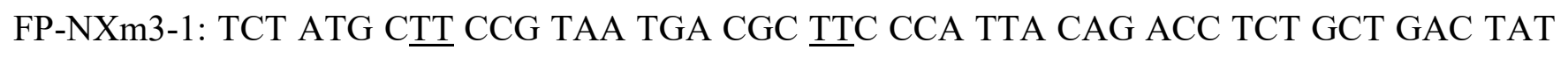
GTG

FP-N4m3-2: TAA TAC GAC TCA CTA TAG GGA GAC CAC AAC GGT TTC CCT GCA TAG

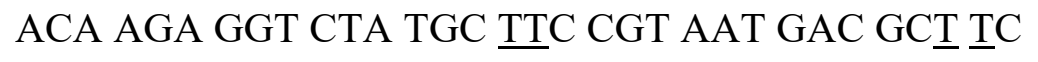

\section{Sequence of pTheo5-PSIV-IRES (p13-2).}

(written from 5' (left) to 3' (right); orange: PSIV IRES; bold: SpeI site)

CTAAATTGTAAGCGTTAATATTTTGTTAAAATTCGCGTTAAATTTTTGTTAAATCAGCTCAT TTTTTAACCAATAGGCCGAAATCGGCAAAATCCCTTATAAATCAAAAGAATAGACCGAGAT 
AGGGTTGAGTGGCCGCTACAGGGCGCTCCCATTCGCCATTCAGGCTGCGCAACTGTTGGGA AGGGCGTTTCGGTGCGGGCCTCTTCGCTATTACGCCAGCTGGCGAAAGGGGGATGTGCTGC AAGGCGATTAAGTTGGGTAACGCCAGGGTTTTCCCAGTCACGACGTTGTAAAACGACGGCC AGTGAGCGCGACGTAATACGACTCACTATAGGGCGAATTGAAGGAAGGCCGTCAAGGCCG CATGAGCTCCATACGATTTAGGTGACACTATAGAACTCACCTATCTCCCCAACACCTAATA ACATTCAATCACTCTTTCCACTAACCACCTATCTACATCACCAAGATATCACTAGTGAAATT AATACGACTCACTATAGGGAGACCACAACGGTTTCCCAGGTATAGACAAGAGGTCTATAC CAGCCGAAAGGCCCTTGGCAGAGACCTCTGCTGACTATGTGATCTTATTAAAATTAGGTTA AATTTCGAGGTTAAAAATAGTTTTAATATTGCTATAGTCTTAGAGGTCTTGTATATTTATAC TTACCACACAAGATGGACCGGAGCAGCCCTCCAATATCTAGTGTACCCTCGTGCTCGCTCA AACATTAAGTGGTGTTGTGCGAAAAGAATCTCACTTCAAGAAAAAGAAACTAGTATGGGC TCCAGCCATCACCATCACCATCATAGCAGCGGCCTGGTGCCGCGCGGCAGCCACATGGGCG GCACTTCCAGCAAGGGCGAGGAGCTGTTCACCGGGGTGGTGCCCATCCTGGTCGAGCTGG ACGGCGACGTGAACGGCCACAAGTTCAGCGTGCGCGGCGAGGGCGAGGGCGATGCCACCA TCGGCAAGCTGACCCTGAAGTTCATCTGCACCACCGGCAAGCTGCCCGTGCCCTGGCCCAC CCTCGTGACCACCCTGACCTACGGCGTGCAGTGCTTCAGCCGCTACCCCGACCACATGAAG CGCCACGACTTCTTCAAGTCCGCCATGCCCGAAGGCTACGTCCAGGAGCGCACCATCTCCT TCAAGGACGACGGCAAGTACAAGACCCGCGCCGTGGTGAAGTTCGAGGGCGACACCCTGG TGAACCGCATCGAGCTGAAGGGCACCGACTTCAAGGAGGACGGCAACATCCTGGGGCACA AGCTGGAGTACAACTTCAACAGCCACAACGTCTATATCACCGCCGACAAGCAGAAGAACG GCATCAAGGCCAACTTCACCGTGCGCCACAACGTGGAGGACGGCAGCGTGCAGCTCGCCG ACCACTACCAGCAGAACACCCCCATCGGCGACGGCCCCGTGCTGCTGCCCGACAACCACTA CCTGAGCACCCAGACCGTGCTGAGCAAAGACCCCAACGAGAAGGGCACCCGCGATCACAT GGTCCTGCACGAGTACGTGAACGCCGCCGGGATCACTTAAGGTACCCTGGGCCTCATGGGC 
CTTCCTTTCACTGCCCGCTTTCCAGTCGGGAAACCTGTCGTGCCAGCTGCATTAACATGGTC ATAGCTGTTTCCTTGCGTATTGGGCGCTCTCCGCTTCCTCGCTCACTGACTCGCTGCGCTCG GTCGTTCGGGTAAAGCCTGGGGTGCCTAATGAGCAAAAGGCCAGCAAAAGGCCAGGAACC GTAAAAAGGCCGCGTTGCTGGCGTTTTTCCATAGGCTCCGCCCCCCTGACGAGCATCACAA AAATCGACGCTCAAGTCAGAGGTGGCGAAACCCGACAGGACTATAAAGATACCAGGCGTT TCCCCCTGGAAGCTCCCTCGTGCGCTCTCCTGTTCCGACCCTGCCGCTTACCGGATACCTGT CCGCCTTTCTCCCTTCGGGAAGCGTGGCGCTTTCTCATAGCTCACGCTGTAGGTATCTCAGT TCGGTGTAGGTCGTTCGCTCCAAGCTGGGCTGTGTGCACGAACCCCCCGTTCAGCCCGACC GCTGCGCCTTATCCGGTAACTATCGTCTTGAGTCCAACCCGGTAAGACACGACTTATCGCC ACTGGCAGCAGCCACTGGTAACAGGATTAGCAGAGCGAGGTATGTAGGCGGTGCTACAGA GTTCTTGAAGTGGTGGCCTAACTACGGCTACACTAGAAGAACAGTATTTGGTATCTGCGCT CTGCTGAAGCCAGTTACCTTCGGAAAAAGAGTTGGTAGCTCTTGATCCGGCAAACAAACCA CCGCTGGTAGCGGTGGTTTTTTTGTTTGCAAGCAGCAGATTACGCGCAGAAAAAAAGGATC TCAAGAAGATCCTTTGATCTTTTCTACGGGGTCTGACGCTCAGTGGAACGAAAACTCACGT TAAGGGATTTTGGTCATGAGATTATCAAAAAGGATCTTCACCTAGATCCTTTTAAATTAAA AATGAAGTTTTAAATCAATCTAAAGTATATATGAGTAAACTTGGTCTGACAGTTATTAGAA AAATTCATCCAGCAGACGATAAAACGCAATACGCTGGCTATCCGGTGCCGCAATGCCATAC AGCACCAGAAAACGATCCGCCCATTCGCCGCCCAGTTCTTCCGCAATATCACGGGTGGCCA GCGCAATATCCTGATAACGATCCGCCACGCCCAGACGGCCGCAATCAATAAAGCCGCTAA AACGGCCATTTTCCACCATAATGTTCGGCAGGCACGCATCACCATGGGTCACCACCAGATC TTCGCCATCCGGCATGCTCGCTTTCAGACGCGCAAACAGCTCTGCCGGTGCCAGGCCCTGA TGTTCTTCATCCAGATCATCCTGATCCACCAGGCCCGCTTCCATACGGGTACGCGCACGTTC AATACGATGTTTCGCCTGATGATCAAACGGACAGGTCGCCGGGTCCAGGGTATGCAGACG ACGCATGGCATCCGCCATAATGCTCACTTTTTCTGCCGGCGCCAGATGGCTAGACAGCAGA 
TCCTGACCCGGCACTTCGCCCAGCAGCAGCCAATCACGGCCCGCTTCGGTCACCACATCCA GCACCGCCGCACACGGAACACCGGTGGTGGCCAGCCAGCTCAGACGCGCCGCTTCATCCT GCAGCTCGTTCAGCGCACCGCTCAGATCGGTTTTCACAAACAGCACCGGACGACCCTGCGC GCTCAGACGAAACACCGCCGCATCAGAGCAGCCAATGGTCTGCTGCGCCCAATCATAGCC AAACAGACGTTCCACCCACGCTGCCGGGCTACCCGCATGCAGGCCATCCTGTTCAATCATA CTCTTCCTTTTTCAATATTATTGAAGCATTTATCAGGGTTATTGTCTCATGAGCGGATACAT ATTTGAATGTATTTAGAAAAATAAACAAATAGGGGTTCCGCGCACATTTCCCCGAAAAGTG CCAC

\section{Sequences of Artificial Riboswitches (nDNA-547-N3/4/5).}

(written from 5' (left) to 3' (right); italic: 5' terminal stem-loop; magenta: MS; [X]: C, GC, or UGC for nDNA-547-N3, nDNA-547-N4, or nDNA-547-N5, respectively; green: aaIRES; blue and red: nDNA547-mini without the end stem; purple: aIRES; orange: PSIV IRES; underlined: start codon; bold: SpeI site)

GGGAGACCACAACGGUUUCCCA[X]AUAGACAAGAGGUCUAUGCAACCGUAAUGACGCAAC CCAUUACAGACCUCUGCUGACUAUGUGAUCUUAUUAAAAUUAGGUUAAAUUUCGAGGU

UAAAAAUAGUUUUAAUAUUGCUAUAGUCUUAGAGGUCUUGUAUAUUUAUACUUACCAC ACAAGAUGGACCGGAGCAGCCCUCCAAUAUCUAGUGUACCCUCGUGCUCGCUCAAACAU UAAGUGGUGUUGUGCGAAAAGAAUCUCACUUCAAGAAAAAGAAACUAGU... 
(A)

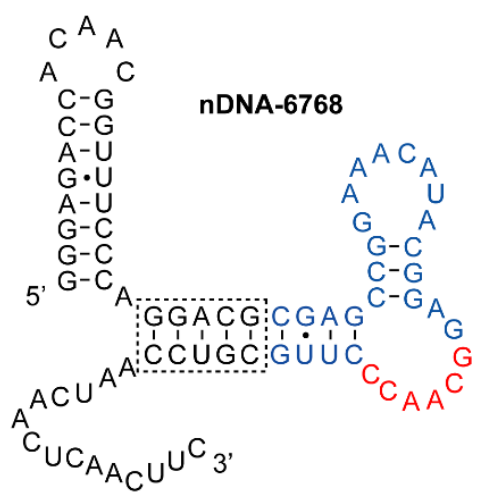

(C)

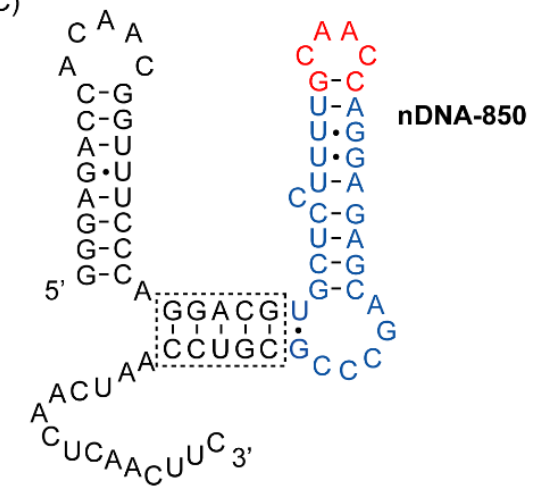

(E)

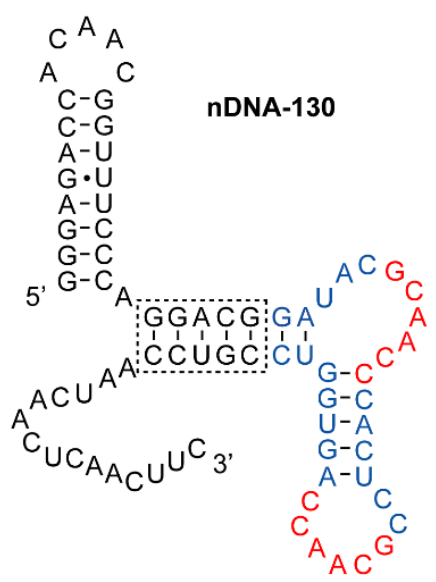

(B)

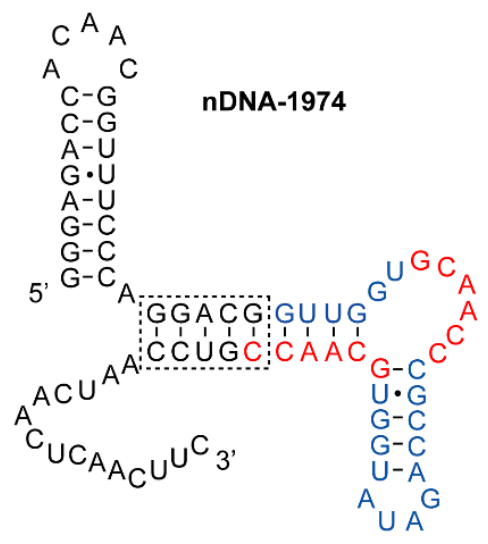

(D)

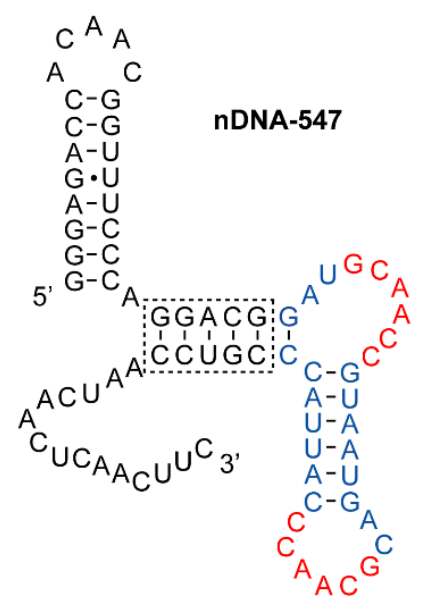

Figure S1. Predicted secondary structures of the selected RNA aptamers, nDNA-6768 (A), nDNA-1974 (B), nDNA-850 (C), nDNA-547 (D), and nDNA-130 (E). They were predicted by RNAstructure software. 
(A)

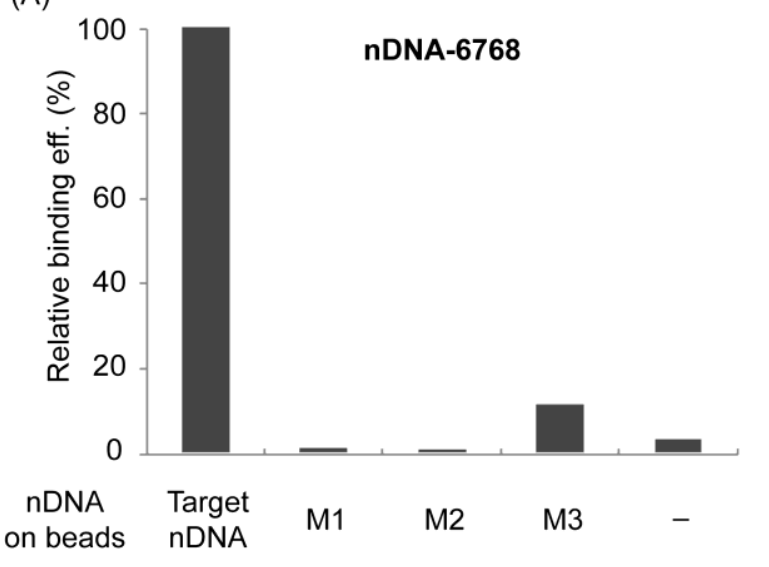

(C)

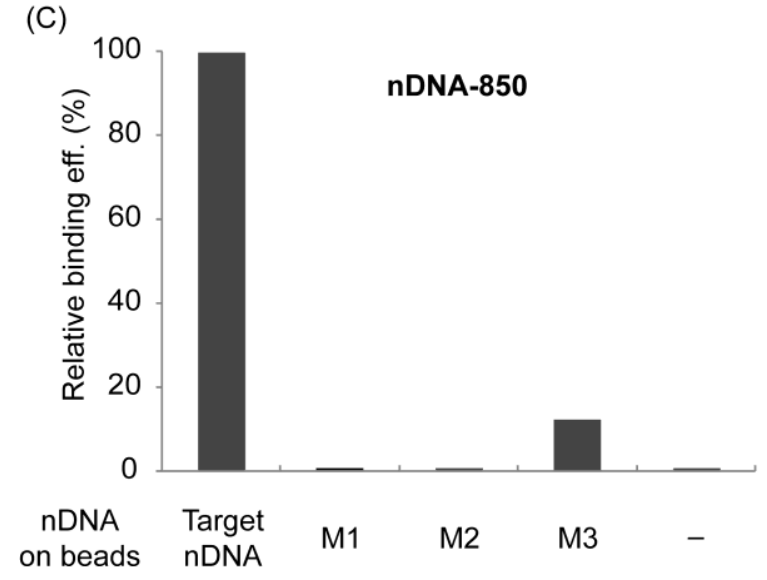

(E)

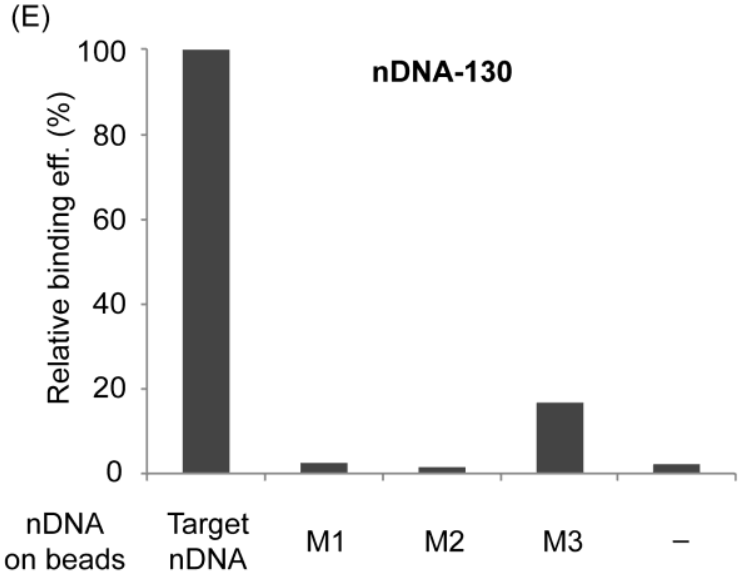

(B)

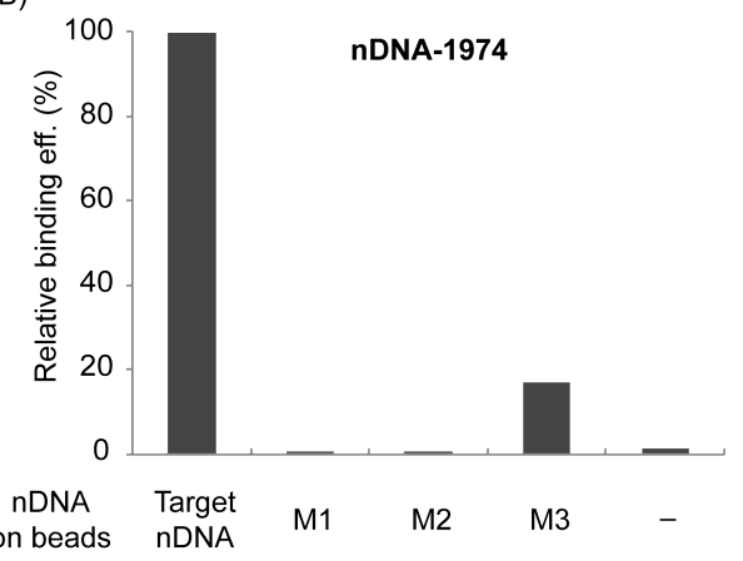

(D)

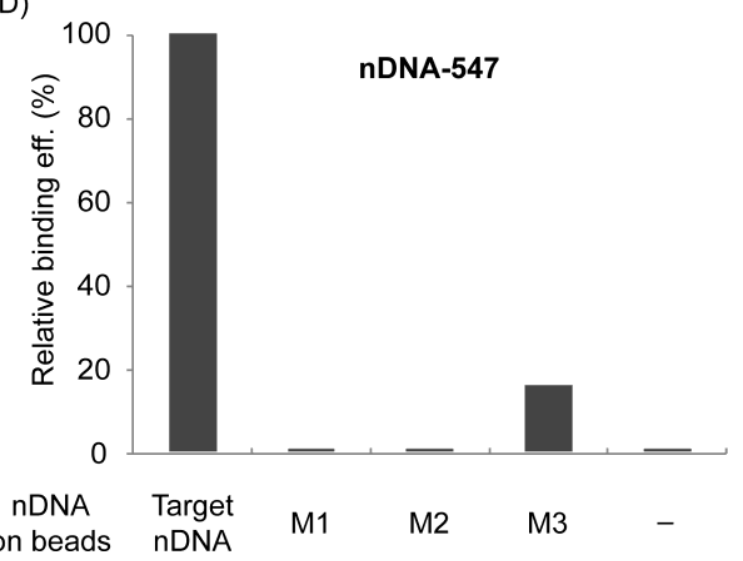

Figure S2. Relative binding efficiency of each RNA aptamer $(1 \mu \mathrm{M})$ against the target nDNA, M1, M2, or M3 $(1 \mu \mathrm{M})$ immobilized on SAv-beads, or the same amount of free beads in the expression buffer. 

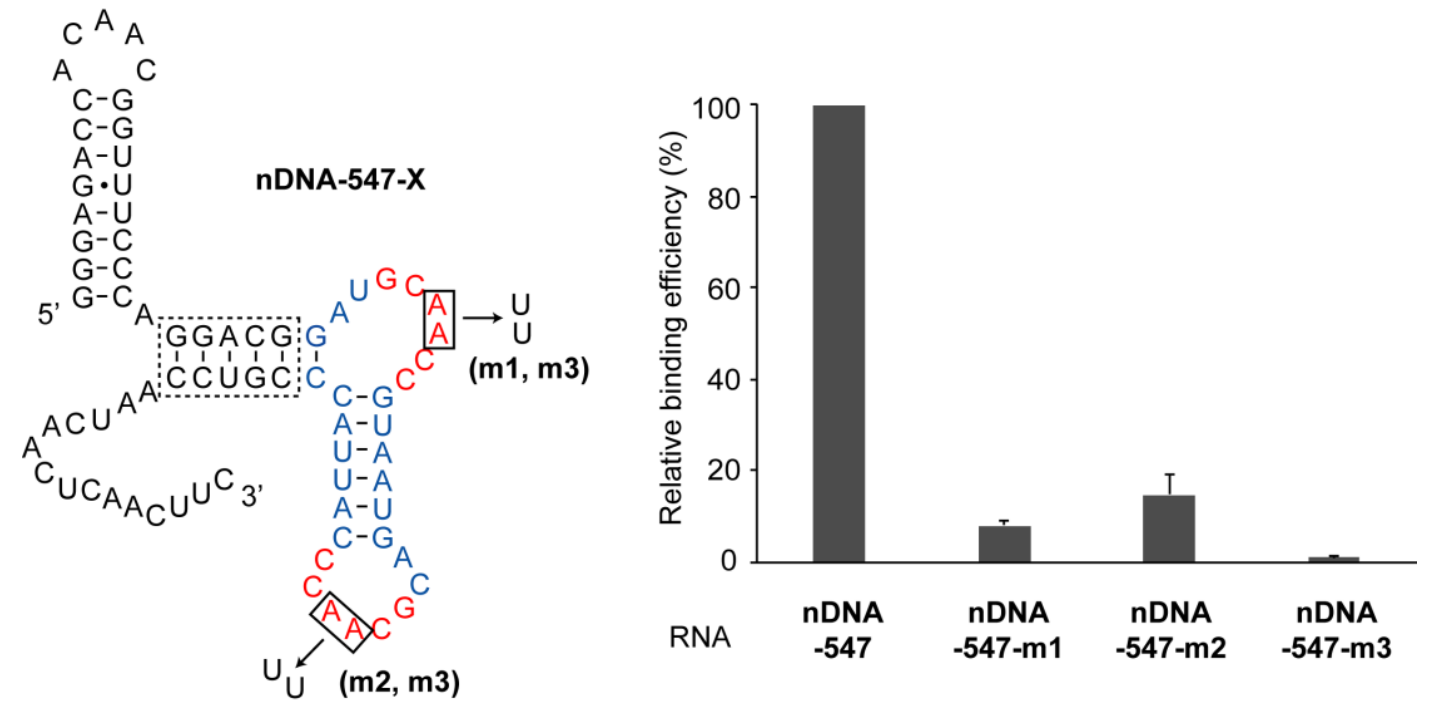

Figure S3. Mutations into CS(s) in nDNA-547 (5' GCAACC 3' to 5' GC $\underline{U U C C}$ 3') and their effects on binding efficiency. Predicted secondary structure of mutants, $\mathbf{n D N A - 5 4 7 - X}$ (left, $\mathbf{X}=\mathbf{m 1}$ : with mutations in the upstream CS; $\mathbf{X}=\mathbf{m} 2$ : with mutations in the downstream $\mathrm{CS} ; \mathbf{X}=\mathbf{m} 3$ : with mutations in both CSs), and relative binding efficiency of each mutant $(1 \mu \mathrm{M})$ against the target nDNA $(1 \mu \mathrm{M})$ immobilized on SAv-beads in the expression buffer (right). 


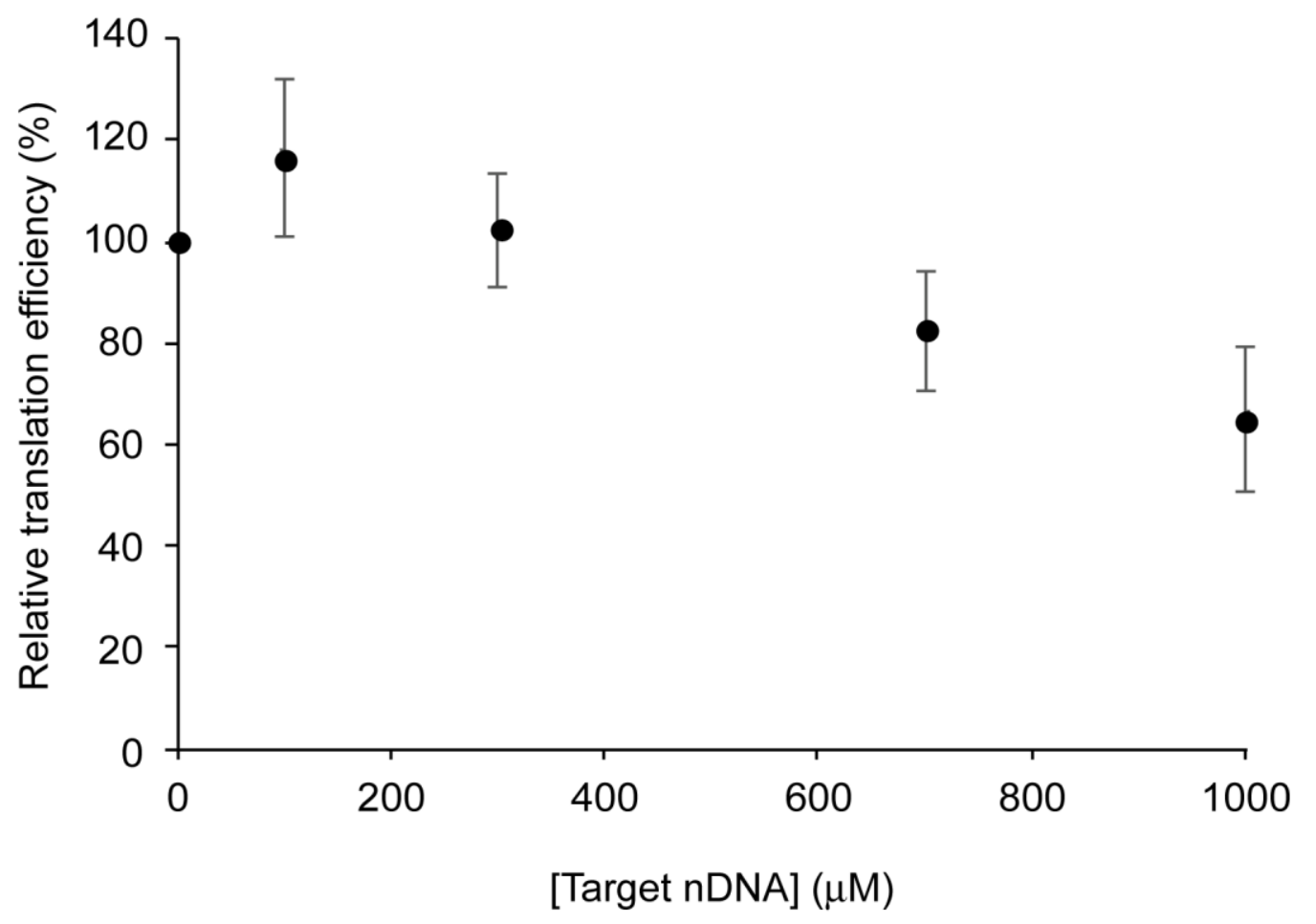

Figure S4. Relative translation efficiencies of the positive control mRNA in the presence of various concentrations of the target nDNA in WGE. 


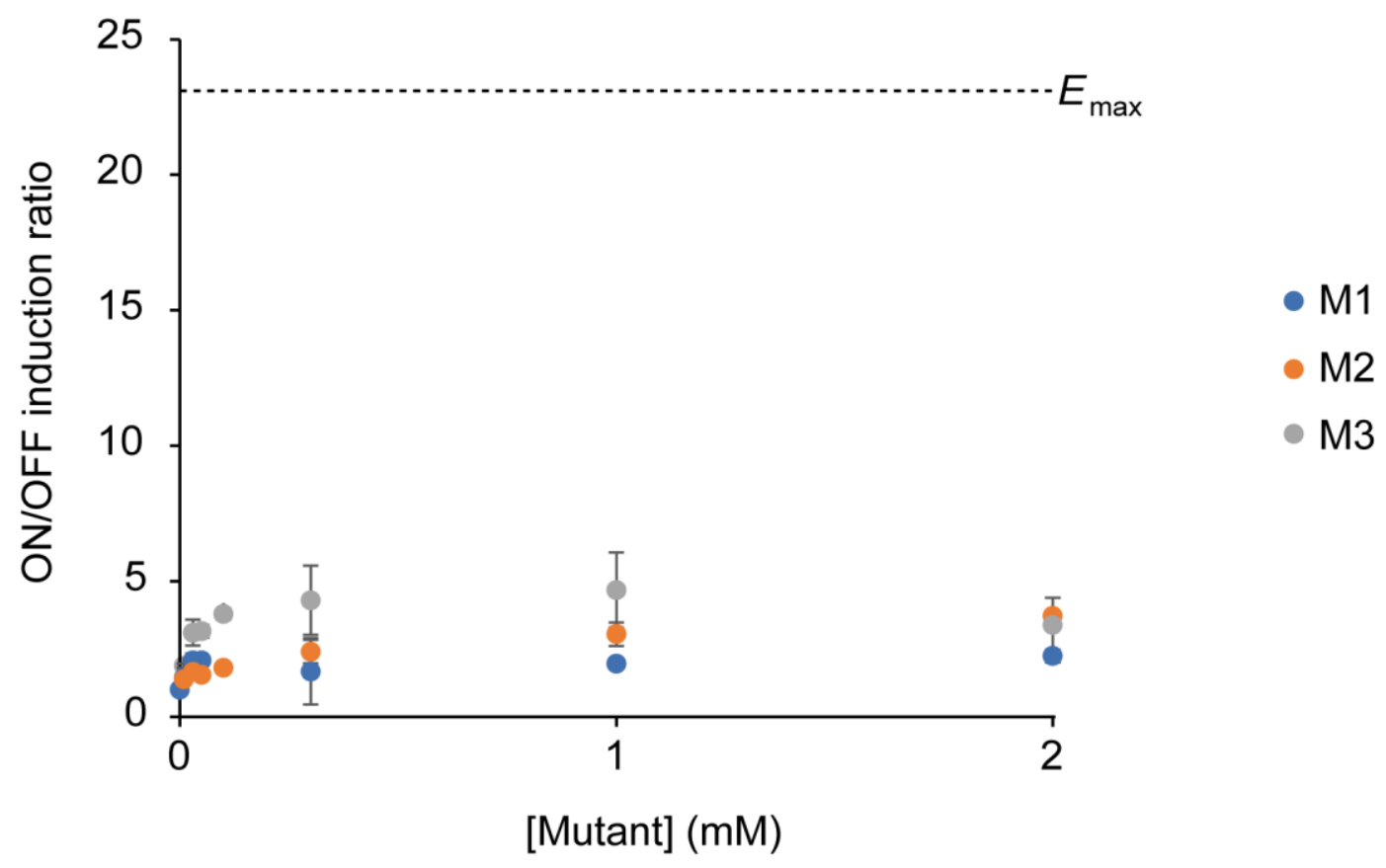

Figure S5. The ON/OFF induction ratios of NanoLuc-encoding mRNA with nDNA-547-N4 at various concentrations of each mutant (M1, M2, or M3). The maximum ON/OFF ratio $\left(E_{\max }\right)$ calculated with the target nDNA is indicated with the dotted line. 
Table S1. Conditions of in vitro selection. Concentrations of RNA in the positive selection were calculated assuming that all of RNA inputted into the previous negative selection were recovered.

\begin{tabular}{|c|c|c|c|c|}
\hline \multirow[b]{2}{*}{ Round } & \multicolumn{2}{|c|}{ Positive selection } & \multicolumn{2}{|c|}{ Counter selection } \\
\hline & $\begin{array}{c}\text { [RNA] } \\
(\mathrm{nM})\end{array}$ & $\begin{array}{c}{[\mathrm{nDNA}]} \\
(\mathrm{nM})\end{array}$ & $\begin{array}{c}\text { Mutant } \\
\text { nDNA }\end{array}$ & $\begin{array}{c}\text { [Mutant] } \\
(\mathrm{nM})\end{array}$ \\
\hline 1 & 2500 & 1000 & - & - \\
\hline 2 & 500 & 250 & - & - \\
\hline 3 & 100 & 50 & - & - \\
\hline 4 & 100 & 50 & - & - \\
\hline 5 & 100 & 50 & - & - \\
\hline 6 & 100 & 50 & - & - \\
\hline 7 & 100 & 50 & - & - \\
\hline 8 & 100 & 50 & M1 & 500 \\
\hline 9 & 100 & 50 & M2 & 500 \\
\hline 10 & 100 & 50 & M3 & 500 \\
\hline 11 & 100 & 50 & $\mathrm{M} 1, \mathrm{M} 2, \mathrm{M} 3$ & 5000 ea \\
\hline 12 & 20 & 10 & $\mathrm{M} 1, \mathrm{M} 2, \mathrm{M} 3$ & 1000 ea \\
\hline 13 & 20 & 10 & $\mathrm{M1}$ 1,M2,M3 & 1000 ea \\
\hline 14 & 20 & 10 & M1,M2,M3 & 1000 ea \\
\hline
\end{tabular}


Table S2. Forward and reverse primers (FP and RP, respectively) used in the sequential PCRs for preparation of mRNAs.

\begin{tabular}{|c|c|c|c|c|c|c|c|c|c|c|c|c|}
\hline \multirow[b]{3}{*}{ Objective mRNA } & \multicolumn{6}{|c|}{5 ' segment } & \multicolumn{4}{|c|}{ 3' segment } & \multirow{2}{*}{\multicolumn{2}{|c|}{$\begin{array}{c}\text { Post-ligation } \\
\text { Final PCR }\end{array}$}} \\
\hline & \multicolumn{2}{|c|}{ 1st PCR } & \multicolumn{2}{|c|}{ 2nd PCR } & \multicolumn{2}{|c|}{ 3rd PCR } & \multicolumn{2}{|c|}{ 1st PCR } & \multicolumn{2}{|c|}{ 2nd PCR } & & \\
\hline & FP & $\mathrm{RP}$ & FP & $\mathrm{RP}$ & FP & $\mathrm{RP}$ & FP & $\mathrm{RP}$ & FP & $\mathrm{RP}$ & FP & $\mathrm{RP}$ \\
\hline Positive control & FP-P-1 & RP-PI & FP-5F & RP-PI & - & - & FP-NL & RP-3U-1 & FP-NL & RP-3U-2 & FP-F & RP-F \\
\hline nDNA-547-N3-NLuc & FP-NX-1 & RP-PI & FP-N3-2 & RP-PI & FP-5F & RP-PI & FP-NL & RP-3U-1 & FP-NL & RP-3U-2 & FP-F & RP-F \\
\hline nDNA-547-N4-NLuc & FP-NX-1 & RP-PI & FP-N4-2 & RP-PI & FP-5F & RP-PI & FP-NL & RP-3U-1 & FP-NL & RP-3U-2 & FP-F & RP-F \\
\hline nDNA-547-N5-NLuc & FP-NX-1 & RP-PI & FP-N5-2 & RP-PI & FP-5F & RP-PI & FP-NL & RP-3U-1 & FP-NL & RP-3U-2 & FP-F & RP-F \\
\hline nDNA-547-N4-m3-NLuc & FP-NXm3-1 & RP-PI & FP-N4m3-2 & RP-PI & FP-5F & RP-PI & FP-NL & RP-3U-1 & FP-NL & RP-3U-2 & FP-F & RP-F \\
\hline
\end{tabular}

